\title{
IMAGING MOLECULAR ENVELOPES OF EVOLVED STARS
}

\author{
JOHN H. BIEGING \\ Steward Observatory, University of Arizona, Tucson AZ 85721 USA
}

\begin{abstract}
Resolved images of molecular emission lines from the envelopes of cool evolved stars reveal much about the physical processes by which such stars lose mass, about the chemical processes which occur in the outflowing gas, and about the evolution of the star to the planetary nebula stage. This paper reviews observational work on molecular line imaging at $\mathrm{mm}$-wavelengths of carbon stars, Miras, proto-planetary nebulae, and planetary nebulae. The impact of these observations on our understanding of the late stages of stellar evolution as it affects the circumstellar environment is emphasized.
\end{abstract}

\section{INTRODUCTION}

The circumstellar envelopes of cool evolved stars are well-suited for investigations with interferometers operating at millimeter and submm wavelengths. The envelopes of such stars are typically molecular in composition and so are detectable in the $\mathrm{mm}$ - and submm-wavelength rotational emission lines of their constituent species. Because the stellar envelopes have relatively small linear dimensions $(<0.1 \mathrm{pc})$ they subtend small angular dimensions for even the nearest known objects. Well-resolved images of molecular line emission must therefore be obtained with interferometric techniques. In this paper I review results of interferometer aperture synthesis work on the molecular envelopes of these stars.

The topic of mass loss from cool evolved stars has been the subject of a number of symposia (see, e.g., Kwok and Pottasch 1987; Mennessier and Omont 1990; Schwarz 1992) and review articles (e.g., Bieging 1988, 1991, 1992) in the past few years, and will not be discussed in detail here. Key aspects of the stellar mass loss phenomenon which molecular line imaging has begun to address include (1) the chemical processes which occur in the stellar envelope; (2) dust formation and grain growth; (3) physical processes affecting mass loss; (4) the nature of post-AGB high-velocity mass ejection; (5) relationship between stellar mass loss and formation of planetary nebulae.

\section{CHEMISTRY OF COOL STAR ENVELOPES}

Mm-wavelength imaging of molecular lines has led to significant progress in understanding the chemical processes in cool star envelopes. The best-studied example is the carbon star IRC +10216 , in which more than 50 molecular species 
have so far been detected (Olofsson 1992). The remarkably high abundances of complex organic species were unexpected, based on the molecules that could form in or just above the photosphere. Over the past ten years, several rather detailed models have been published which invoke photochemical processes to explain the large abundances of organic molecules in this object (Glassgold et al. 1986, 1987; Nejad and Millar 1987; Howe and Millar 1990; Cherchneff et al. 1993). The basic idea of these models is that gas phase reactions are initiated by UV photons from the ambient interstellar radiation field, and also from cosmic rays, by the production of molecular ions and neutral radicals. These reactive species combine with the abundant, stable molecules like $\mathrm{CO}, \mathrm{HCN}$, and $\mathrm{C}_{2} \mathrm{H}_{2}$, which are formed in the photosphere and carried out in the wind. The result of these reactions is the production of a variety of complex organic molecules, most of which should be distributed in shells surrounding the star. In contrast, molecules produced in the photosphere should have nearly constant abundances out to a distance where the dusty stellar envelope becomes sufficiently transparent to UV photons that photodissociation and reactions with ions or radicals destroy the molecules of photospheric origin, at which point their abundances are predicted to fall sharply.

The first aperture synthesis images of a mm-wavelength molecular emission line toward IRC +10216 were of the $3.4 \mathrm{~mm} \mathrm{~J}=1-0$ line of $\mathrm{HCN}$ (Bieging, Chapman and Welch 1984) and were obtained with the U.C. Berkeley interferometer at Hat Creek. These images confirmed that the HCN distribution is cut off at about the radius predicted by photochemical models. A detailed statistical equilibrium analysis of the images indicated that the density of $\mathrm{HCN}$ molecules deviated by about a factor of 3 from the $1 / \mathrm{r}^{2}$ behavior expected in a constant abundance, constant mass loss rate outflow. This variation in HCN abundance suggested possible variations with time of the mass loss rate of the envelope chemical composition (e.g., because of a change in the photospheric $\mathrm{C} / \mathrm{O}$ ratio). Subsequent observations of IRC +10216 in lines of $\mathrm{HC}_{3} \mathrm{~N}, \mathrm{C}_{2} \mathrm{H}$, and $\mathrm{HNC}$ by Bieging and Nguyen-Q.-Rieu (1988a) succeeded in resolving the predicted shell structure for those molecules. Based on a simple LTE analysis they calculated abundance distributions for the 3 molecules as a function of distance from the star. For $\mathrm{HNC}$ and $\mathrm{C}_{2} \mathrm{H}$, the agreement with predictions of photochemical models was in fact rather good. This agreement gave credibility to the chemical models, which depended on ion-molecule reactions. For $\mathrm{HC}_{3} \mathrm{~N}$, however, there was a serious discrepancy, in that the peak abundance inferred from the observations was 1 to 2 orders of magnitude larger than predicted by the ion-molecule photochemical models. In a more recent study, Bieging and Tafalla (1993) presented images of $\mathrm{HC}_{3} \mathrm{~N}, \mathrm{C}_{3} \mathrm{~N}$, and $\mathrm{SiS}$ emission at $\sim 109 \mathrm{GHz}$. Their analysis of the $\mathrm{HC}_{3} \mathrm{~N}$ data also showed that the molecular abundance was much larger than predicted by the ion-molecule reaction models. This discrepancy has prompted a re-evaluation of the importance of another class of chemical reactions-those between neutral radicals and the abundant stable photospheric species like HCN and $\mathrm{C}_{2} \mathrm{H}_{2}$. Model calculations by Howe and Millar (1990) and Cherchneff, Glassgold, and Mamon (1993) which included neutral radical reactions such as $\mathrm{CN}$ $+\mathrm{C}_{2} \mathrm{H}_{2} \rightarrow \mathrm{HC}_{3} \mathrm{~N}+\mathrm{H}$, produced a much better fit to the $\mathrm{HC}_{3} \mathrm{~N}$ abundance distribution than the earlier ion-molecule photochemical models. The resolved molecular line images provide a very strong test of the chemical models, because these models make quite specific predictions about the radial abundance varia- 
tions of each molecular species, and about the relative locations and abundances of related species like $\mathrm{HC}_{3} \mathrm{~N}$ and $\mathrm{C}_{3} \mathrm{~N}$. Such predictions can only be tested by image data, not by unresolveed or poorly-resolved single-dish spectroscopy.

Another molecular species recently mapped by aperture synthesis techniques is the simple ring molecule $\mathrm{SiC}_{2}$. Images obtained by Gensheimer, Likkel, and Snyder (1992) with the BIMA interferometer showed that emission from the 95.579 $\mathrm{GHz}$ line was distributed in what appeared to be a broken, asymmetric shell. A similar study by Takano, Saito, and Tsuji (1992) of the $93.064 \mathrm{GHz}$ line of $\mathrm{SiC}_{2}$ with the Nobeyama Millimeter Array showed a clear shell-like structure with a diameter of about $30^{\prime \prime}$. They also found that the $\mathrm{SiC}_{2}$ distribution was clumpy and asymmetric, with some indications of a bipolar morphology. Takano et al. (1992) demonstrated that their observed distribution of $\mathrm{SiC}_{2}$ is inconsistent with a photspheric origin for the molecule. It must be produced by photochemical reactions in the outer envelope. Calculations by Glassgold et al. (1986) and Glassgold and Mamon (1992) show that $\mathrm{SiC}_{2}$ can be produced by ion-molecule reactons in the outer envelope. The model of Glassgold and Mamon is in qualitative agreement with the observations, although molecular abundances have not been derived from the imaging data as yet. Even so, the observed distribution clearly implies that $\mathrm{SiC}_{2}$ is generated by photochemical processes at distances of a few $\times 10^{16} \mathrm{~cm}$ from the star.

\section{GAS-GRAIN PROCESSES}

The formation and growth of dust grains in red giant winds is of great interest, since these winds may be a major source of the refractory component of interstellar dust (cf. Jura 1987). Observations of molecular lines can give information about the growth of dust grains by revealing the loss of refractory elements from the gas phase in the wind. Probably the most readily-studied refractory element is silicon. Although some $\mathrm{Si}$ remains in the gas phase at large distances from IRC +10216 , as the images just discussed of $\mathrm{SiC}_{2}$ clearly show, these molecules must contain only a small fraction of the total amount of Si present. For carbon stars like IRC+10216, the dominant gas phase form of $\mathrm{Si}$ is expected to be $\mathrm{SiS}$, produced in the photosphere and carried out in the wind (Lafont et al. 1982).

There have been two imaging studies of the distribution of SiS in the envelope of IRC+10216. Bieging and Nguyen-Q.-Rieu (1989) mapped the J=5-4 and 6-5 lines with the BIMA array and, from an excitation analysis, concluded that the SiS abundance drops by a factor of at least 10 from the inner envelope to $\sim 4 \times 10^{16} \mathrm{~cm}$ and is cut off by photodissociation at about $8 \times 10^{16} \mathrm{~cm}$ (all for an assumed distance of $200 \mathrm{pc}$ ). In a related study, Bieging and Tafalla (1993) obtained higher resolution $\left(5^{\prime \prime}\right)$ images of the $J=6-5$ line and performed a detailed statistical equilibrium analysis. They also concluded that the $\mathrm{SiS}$ abundance must drop rapidly at distances of a few $\times 10^{15} \mathrm{~cm}$ from the star and must be cut off by photodissociation. The removal of SiS from the gas phase is thought to be a result of grain adsorption processes, so that the refractory element $\mathrm{Si}$ is incorporated into the solid particles. Details of this process (and why Si, but not $\mathrm{S}$, is strongly depleted) are far from understood. However, a simple model by Glassgold and Mamon (1992) in which SiS is removed from the gas by adsorption onto grains, predicts a qualitatively similar behavior in the SiS abundance 
as a function of radius. The model calculations indicate that the shape of the abundance function in the inner regions depends on a "grain sticking" coefficient which determines the rate at which SiS molecules are adsorbed. In principal, the SiS abundance versus radius derived from observations should constrain the values of this parameter, which is in turn related to the microscopic physics of the adsorption process.

In the case of stars with $\mathrm{C} / \mathrm{O}<1$, i.e., the M-giants, the principal form of silicon in the gas phase is predicted to be SiO. M-giants are frequently found to be sources of $\mathrm{SiO}$ maser emission, as well as non-maser emission. As in the case of $\mathrm{SiS}$ in carbon star envelopes, the distribution of $\mathrm{SiO}$ can be used to constrain models for the process of grain growth. In a study of the $J=2-1 \mathrm{v}=0$ (non-maser) emission line of $\mathrm{SiO}$ at $86.85 \mathrm{GHz}$ Lucas et al. (1992) used the IRAM interferometer to make "snapshot" (i.e., short integration) images of $10 \mathrm{M}$ - stars, $1 \mathrm{~S}$-star, and 1 carbon star. They determined the size of the SiO emission as a function of observed radial velocity by fitting model visibilities for an assumed exponential brightness distribution. Surprisingly, the size of the distribution hardly varied with velocity for all but 2 of the stars. This behavior is in contrast to that expected for resolved images of a uniformly expanding, constant abundance envelope, where the size of the emission should vary across the spectral line, with a maximum size at the central velocity and minima at the velocity extremes. On the basis of an LTE model with a constant SiO abundance throughout the envelope, they conclude that their observations imply that the stellar winds for their sample of stars must still be accelerated at distances of several $\times 10^{15} \mathrm{~cm}$ from the star. A further implication is that significant grain growth must occur at distances as large as several $\times 10^{15} \mathrm{~cm}$. Lucas et al. (1992) note that these conclusions are seriously at variance with conventional theoretical ideas about wind acceleration and grain growth in circumstellar envelopes.

Sahai and Bieging (1993) reached an alternative conclusion in a closely related study. Using the BIMA array, they made images of the same $\mathrm{SiO} \mathrm{J}=2-1$ line toward two M-giants (IK Tau and RX Boo) and one S-star ( $\chi$ Cyg). They found that the $\mathrm{SiO}$ emission is strongly centrally peaked but has an extended low-brightness component. Like Lucas et al. (1992) they found that the apparent size of the $\mathrm{SiO}$ emission is nearly constant with velocity across the spectral line. Sahai and Bieging performed a statistical equilibrium/radiative transfer model analysis and concluded that, contrary to the conclusions of Lucas et al. (1992), the SiO brightness distributions could be explained by a wind with a constant outflow velocity but decreasing relative $\mathrm{SiO}$ abundance with increasing distance from the star. Their models predict a power-law variation of $\mathrm{SiO}$ line brightness with projected distance from the star, of the form $T_{B} \sim r^{-1.5}$. The model analysis of Sahai and Bieging requires that the $\mathrm{SiO}$ abundance falls rapidly for distances out to $\sim 1-2 \times 10^{15} \mathrm{~cm}$, and much more slowly out to the photodissociation cutoff at $\sim 1 \times 10^{16} \mathrm{~cm}$. The initial rapid decrease in gasphase $\mathrm{SiO}$ in the outflowing wind is presumably a result of adsorption onto dust grains. Sahai and Bieging (1993) argue that, in contrast to Lucas et al. (1992), the interferometer images of $\mathrm{SiO}$ emission do not require any unusual wind acceleration at large distances, but rather are consistent with a conventional wind velocity law and a decrease in gas phase $\mathrm{SiO}$ abundance as a consequence of grain adsorption, which contributes to the growth of silicate grains. 


\section{EVOLUTION TO PLANETARY NEBULAE}

The processes by which an AGB star is transformed to a hot stellar remnant surrounded by a planetary nebula (PN) are not well understood. Molecular line imaging by mm-interferometry can examine several aspects of these processes. Important issues include: (1) the nature of a "superwind" mechanism leading to high mass loss rates and/or very high velocity outflows; (2) the hydrodynamics of interactions between a fast wind overtaking a previous slow wind; (3) development of non-spherical geometry, notably the bipolar morphology often found in transition objects; (4) effects of the central star (with $T_{\text {eff }} \geq 10^{5} \mathrm{~K}$ ) on the neutral envelope, including photo-ionization and -dissociation leading to observable chemical effects. In the last several years, a number of interferometric studies have begun to address these questions.

An excellent example of an object believed to be in the transition phase from AGB star to PN is CRL 2688 (the "Egg Nebula"). An imaging study of the CO J=1-0 line by Heiligman et al. (1986) with the OVRO interferometer showed that the $\mathrm{CO}$ was distributed over a region slightly larger than the optical lobes, but found no evidence for the bipolar structure which is so remarkable in optical images. This object is very carbon rich and has strong HCN line emission. An imaging study of the $\mathrm{J}=1-0$ line by Bieging and Nguyen-Q.-Rieu (1988b) with the BIMA interferometer showed that the HCN emission is strongly concentrated on the position of the star and has extensions orthogonal to the bipolar axis, consistent with a high-density toroidal gas distribution. They also found a significant gradient in the HCN line velocity centroid which indicated that the general outflow from the star was faster along the bipolar axis and had a significant rotational component about the bipolar axis. They argued that this rotational motion of the wind implied a transport of angular momentum from the stellar system, at a rate so large as to require the presence of a close binary companion. They speculated that the bipolar morphology was a consequence of enhanced mass loss in the orbital plane, perhaps as a result of a magneticallydriven centrifugal wind.

Other recent studies of CRL 2688 in emission lines of $\mathrm{HC}_{5} \mathrm{~N}$ and SiS have been made with the IRAM interferometer and are reported in the review by Lucas elsewhere in this volume.

Another example of a transition object, but at a more advanced stage of evolution, is CRL 618. This star is surrounded by a compact ionized region, within a more extended molecular envelope. Its optical morphology is bipolar, but this is evidently dominated by scattered light from low-density regions. Shibata et al. (1992a) have mapped the CO J=1-0 emission at $3.5^{\prime \prime}$ resolution with the Nobeyama array. They find that the emission is strongly concentrated toward the ionized core, with no indication of bipolarity or of a toroidal distribution of gas perpendicular to the optical lobes. Meixner and Welch (1992) also mapped the $\mathrm{CO} J=1-0$ line with the BIMA array with $3.5^{\prime \prime}$ resolution. They find an unresolved core of $\mathrm{CO}$ emission surrounded by an envelope of about 10 arcsec in size. There is no clear indication that the molecular gas has a distribution related to the optical lobes. The total extent of the molecular envelope is evidently much larger than the core region, according to single dish maps by Phillips et al. (1992) and Bachiller et al. (1989), so that the interferometer images are probably missing some large-scale structure. A fully-sampled image 
of CRL 618 in CO J=1-0 will most probably require a combination of single-dish and interferometer images.

CRL 618 is notable for the very high velocity gas present in its envelope. CO J=2-1 and 3-2 spectra (Gammie et al. 1989; Cernicharo et al. 1989) show wings out to $\pm 200 \mathrm{~km} / \mathrm{s}$, making this one of the fastest outflow sources known. In a study of the HCN J=1-0 emission, Neri et al. (1992) used the IRAM interferometer to resolve the high velocity flow. From their observations, they propose a model in which a high velocity stellar wind impacts slow-moving clumps in the precursor AGB star envelope. The resulting shocks heat and accelerate the clumps, destroying any molecules present. After passage of the shock, HCN and other species quickly form again and produce the observed highvelocity emission. Although rather speculative at present, this picture suggests the potential importance of high resolution spectral line imaging to understand the origin of very high velocity gas in such very young $\mathrm{PN}$.

The young PN NGC 7027 has been imaged interferometrically in emission lines of $\mathrm{CO}$ and $\mathrm{HCO}^{+}$. In an early study with the OVRO interferometer, Masson et al. (1985) mapped the distribution of the CO $\mathrm{J}=1-0$ line and concluded that the molecular gas could be described by an expanding ellipsoidal density distribution with a morphology related to that of the ionized nebula. Bieging, Wilner, and Thronson (1991) combined BIMA interferometer observations with NRAO 12-m single dish maps to obtain CO 1-0 images without the missing flux which affected the Masson et al. results. They found that the $\mathrm{CO}$ distribution is clumpy, with an inner ring whose detailed appearance closely resembles that of the ionized gas, indicating a causal relationship between precursor AGB star wind and PN morphology. In a related study, Graham et al. (1992) present high resolution images of $\mathrm{CO} 1-0$ in the interface region between the ionized gas and molecular envelope of NGC 7027. From $\mathrm{CO}$ kinematics they find that the observed $\mathrm{H}_{2}$ emission is excited by UV photons, not shocks. From a highresolution study of $\mathrm{HCO}^{+} \mathrm{J}=1-0$ emission with the Nobeyama array, Deguchi et al. (1992) conclude that the interface region is ionized by soft X-rays from the central star, resulting in ion-molecule reactions to produce $\mathrm{HCO}^{+}$. They model the $\mathrm{HCO}^{+}$interface region as an expanding torus at the periphery of the ionized gas.

Finally, two other young PN have been mapped in the CO 1-0 line with the Nobeyama array. IRAS $21282+5050$ shows a non-circular CO distribution which Shibata et al. (1989) interpret as an expanding torus of gas. This object closely resembles NGC 7027, they argue, but is in an earlier evolutionary stage. A similar study of M1-7 by Shibata et al. (1992b) resolved the molecular emission into two peaks lying perpendicular to the optical axis, and surrounding the ionized gas. The kinematics of the CO suggest an expanding torus which will develop into a bipolar PN.

\section{ACKNOWLEDGMENTS}

I thank Drs. A. E. Glassgold and J.R. Graham and Ms. M. Meixner for communicating results in advance of publication. This work was supported in part by NSF grant AST-9115137. 


\section{REFERENCES}

Alcock, C., and Ross, R.R. 1986, $A p J, 310,838$

Bachiller, R., Gomez-Gonzalez, J., Bujarrabal, V., and Martin-Pintado, J. 1988, $A \mathcal{E} A, 196, \mathrm{~L} 5$

Bieging, J.H. 1988, PASP, 100, 97

1991, in Atoms, Ions, and Molecules: New Results in Spectral Line Astrophysics, eds. A. Haschick and P. Ho, Astron. Soc. Pacific. Conf. Ser., Vol. 16, p. 95 1992, Advan. in Space Res., in press

Bieging, J.H., Chapman, B., and Welch, W.J. 1984, ApJ, 285, 656

Bieging, J.H., and Nguyen-Q.-Rieu 1988a, $A p J, 329, \mathrm{~L} 107$ 1988b, ApJ, 324, 516 1989, $A p J, 343, \mathrm{~L} 25$

Bieging, J.H., and Tafalla, M. 1993, $A J$, in press

Bieging, J.H., Wilner, D., and Thronson, H.A. 1991, ApJ, 379, 271

Cernicharo, J., Guélin, M., Martin-Pintado, J., Peñalver, J., and Mauersberger, R. $1989, A \mathcal{E} A, 222, \mathrm{~L} 1$

Cherchneff, I., Glassgold, A.E., and Mamon, G.A. 1993, $A p J$, in press

Deguchi, S., Izumiura, H., Nguyen-Q.-Rieu, Shibata, K., Ukita, N., and Yamamura, I. 1992, $A p J, 392,597$

Gensheimer, P.D., Likkel, L., and Snyder, L.E. 1992, ApJ, 388, L31

Gammie, C., Knapp, G., Young, K., Phillips, T., and Falgarone, E. 1989, ApJ, 345, L87

Glassgold, A., Lucas, R., and Omont, A. 1986, $A \mathcal{E A}, 157,35$

Glassgold, A., Mamon, G., Omont, A., and Lucas, R. 1987, $A \& A$, 180, 183

Glassgold, A., and Mamon, G. 1992, in Chemistry and Spectroscopy of Interstellar Molecules, ed. D. Böhme, E. Herbst, N. Kaifu, and S. Saito, Univ. of Tokyo Press, p. 261

Graham, J., et al. 1992, $A J$, in press

Heiligman, G.M., et al. 1986, $A p J, 308,306$

Howe, D., and Millar, T. 1990, MNRAS, 244, 444

Jura, M. 1987, in Interstellar Processes, eds. D.J. Hollenbach and H.A. Thronson, Jr. (Dordrecht: Reidel), p. 3

Kwok, S., and Pottasch, S.R. (eds.) 1987, Late Stages of Stellar Evolution, Dordrecht: Reidel

Lafont, S., Lucas, R., and Omont, A. 1982, $A \mathcal{B} A, 106,201$

Lucas, R., et al. 1992, $A \& A, 262,491$

Masson, C.R., et al. $1985 A p J, 292,464$ 
Meixner, M., and Welch, W.J. 1992, in preparation

Mennessier, M., and Omont, A. (eds.) 1990, From Miras to Planetary Nebulae: Which Path for Stellar Evolution?, Gif-sur-Yvette: Editions Frontieres

Nejad, L., and Millar, T. 1987, $A \mathscr{E} A, 183,279$

Neri, R., Garcia-Burillo, S., Guélin, M., Cernicharo, J., Guilloteau, S., and Lucas, R. 1992, $A \& A, 262,544$

Olofsson, H. 1992, in Schwarz, op cit.

Phillips, J., Williams, P., Mampaso, A., and Ukita, N. 1992, $A \mathscr{G} A, 260,283$

Sahai, R., and Bieging, J.H. 1993, $A J$, in press

Schwarz, H. (ed.) 1992, Mass Loss on the AGB and Beyond, ESO, in press

Shibata, K., Tamura, S., Deguchi, S., Hirano, N., Kameya, O., and Kasuga, T. 1989, $A p J, 345, \mathrm{~L} 55$

Shibata, K., Deguchi, S., Hirano, N., Kameya, O., and Tamura, S. 1992a, ApJ, in press

Shibata, K., Deguchi, S., Kasuga, T., Tamura, S., Hirano, N., and Kameya, O. $1992 \mathrm{~b}$, in IAU Symp. No. 155: Planetary Nebulae, in press

Takano, S., Saito, S., and Tsuji, T. 1992, PASJ, 44, 469

\section{DISCUSSION}

N. Scoville: Does one ever see variations in the abundances of molecules which are photodissociation products between different sides of the star which could be attributed to anisotropy of the interstellar UV? Some molecular images show weak asymmetries that might be attributable to anisotropies in the interstellar UV, but the observed deviations are not large and may be consistent with random or systematic noise, so the evidence for such an effect is not strong.

M. Hayashi: I can see many small fragments in the $H C_{3} N$ map you presented. Are these fragments real? If they are, what do you think about their formation? Most molecular line images of IRC+10216 show such structure in maps of narrow velocity channels. However, there is seldom a good correlation in these clumps from one molecular species to another, which suggests that most of the "structure" is a result of small phase calibration errors and relatively low signal to noise ratios. One should note, though, that the mass loss process is not well understood and may be episodic or stochastic in nature-that is, that blobs of matter are ejected randomly from the star so that the envelope only appears round and symmetric when averaged over all velocities. In M-giants, images of $\mathrm{SiO}$ and $\mathrm{OH}$ maser emission show fragmentary structure which is real and supports the stochastic mass ejection idea (cf. Alcock and Ross 1986). 